\title{
Exosomes from differentially activated macrophages influence dormancy or resurgence of breast cancer cells within bone marrow stroma
}

\author{
Nykia D. Walker ${ }^{1,2}$, Michael Elias ${ }^{1}$, Khadidiatou Guiro ${ }^{1}$, Ranvir Bhatia ${ }^{1}$, Steven J. Greco ${ }^{1}$, Margarette Bryan ${ }^{1}$, \\ Marina Gergues ${ }^{1,2}$, Oleta A. Sandiford ${ }^{1,2}$, Nicholas M. Ponzio ${ }^{3}$, Samuel J. Leibovich ${ }^{4}$ and Pranela Rameshwar ${ }^{1,2}$
}

\begin{abstract}
Breast cancer (BC) cells (BCCs) can retain cellular quiescence for decades, a phenomenon referred to as dormancy. BCCs show preference for the bone marrow (BM) where they can remain dormant for decades. Targeting BCCs within the BM is a challenge since the dormant BCCs reside within BM stroma, also residence for hematopoietic stem cells (HSCs). Dormant BCCs could behave as cancer stem cells (CSCs). The CSCs and HSCs are similar by function and also, by commonly expressed genes. The method by which dormant BCCs transition into clinically metastatic cells remains unclear. This study tested the hypothesis that macrophages (MФs) within BM stroma, facilitates dormancy or reverse this state into metastatic cells. MФs exhibiting an M2 phenotype constitute 10\% of cultured BM stroma. The M2 MФs form gap junctional intercellular communication (GJIC) with CSCs, resulting in cycling quiescence, reduced proliferation and carboplatin resistance. In contrast, MФs expressing the M1 phenotype reversed BC dormancy. Activation of M2a MDs via the toll-like receptor 4 (TLR4) switched to M1 phenotype. The switch can occur by direct activation of M2a MDs, or indirectly through activation of mesenchymal stem cells. M1 MФ-derived exosomes activated NFKB to reverse quiescent BCCs to cycling cells. Using an in vivo model of BC dormancy, injected Mi MOs sensitized BCCs to carboplatin and increased host survival. In summary, we have shown how BM stromal MФs, through exosomes, regulate the behavior of BCCs, by either inducing or reversing dormancy.
\end{abstract}

\section{Introduction}

Breast cancer $(\mathrm{BC})$ cells (BCCs) may exist in cellular quiescence (dormancy) for decades ${ }^{1,2}$. Disseminated $\mathrm{BCCs}$ can enter the bone marrow (BM) long before detection $^{3,4}$. This allows for the establishment of $\mathrm{BC}$ dormancy before clinical diagnosis, in addition to transition into cellular quiescence during the clinical course of the disease ${ }^{5-7}$. As compared to micrometastasis in sentinel lymph nodes, $\mathrm{BC}$ metastasis to the $\mathrm{BM}$ leads to a

\footnotetext{
Correspondence: Pranela Rameshwar (rameshwa@njms.rutgers.edu) ${ }^{1}$ Rutgers New Jersey Medical School (NJMS), Newark, NJ, USA

${ }^{2}$ Rutgers Graduate School of Biomedical Sciences, Newark, NJ, USA Full list of author information is available at the end of the article. Edited by $Y$. Shi
}

worse prognosis ${ }^{8}$. BM stromal cells form a critical niche for BCCs to survive. The stromal cells facilitate BCC quiescence, immune escape, changes in cytokine production and gap junctional intercellular communication $(\mathrm{GJIC})^{9,10}$.

Precise targeting of dormant BCCs in BM is a challenge. The quiescent BCCs have stem cell-like properties, and share similarities with endogenous hematopoietic stem cells (HSCs). The anatomical location of the cancer cells with HSCs makes it difficult to target the dormant BCCs without untoward effects on the hematopoietic system ${ }^{10}$. Nonetheless, an understanding of how BM stroma support $\mathrm{BCC}$ dormancy is important since the same stromal cells can also cause BC resurgence ${ }^{11-13}$. BM stroma is

\section{(c) The Author(s) 2019}

(c) (i) Open Access This article is licensed under a Creative Commons Attribution 4.0 International License, which permits use, sharing, adaptation, distribution and reproduction cc in any medium or format, as long as you give appropriate credit to the original author(s) and the source, provide a link to the Creative Commons license, and indicate if changes were made. The images or other third party material in this article are included in the article's Creative Commons license, unless indicated otherwise in a credit line to the material. If material is not included in the article's Creative Commons license and your intended use is not permitted by statutory regulation or exceeds the permitted use, you will need to obtain permission directly from the copyright holder. To view a copy of this license, visit http://creativecommons.org/licenses/by/4.0/. 
comprised of several cell types such as macrophages (MФs), fibroblasts, osteoblasts, mesenchymal stem cells (MSCs), and adipocytes ${ }^{13,14}$.

MФs are broadly divided into nonactivated, classically activated (M1) and alternatively activated (M2) types ${ }^{15-17}$. M2 MФs are classified as M2a, M2b, M2c, or M2d and such designation, depends on the mode of activation ${ }^{16}$. M1 MФs elicit a proinflammatory response and M2 MФs, immune suppression, wound healing, and angiogenesis ${ }^{17}$. The biological function of a particular $\mathrm{M} \Phi$ type may be influenced by the surrounding niche, such as MSCs within $\mathrm{BM}^{14,18}$. We tested the hypothesis that activation of stromal cells causes one of its component, M2 MФ, to polarize into the M1 phenotype to reverse dormant BCCs into proliferating cells.

This study activated toll-like receptor 4 (TLR4) on MФs to study how this influence BC behavior because TLR4 has been linked to cancer recurrence ${ }^{19-21}$. TLR4 is a member of the pattern recognition receptor (PRR) system, which can be stimulated by microbiome-derived ligands such as lipopolysaccharide (LPS). TLR4 can also bind to other pathogen associated molecular pattern and endogenous damage-associated molecular patterns (DAMPs) ${ }^{22}$.

We report on conversion of M2 MФs into M1 MФ phenotype by LPS. Such conversion occurred directly on M2 MФs and indirectly, through MSCs. The M1 MФs secrete exosomes, which reversed the quiescent phase of BCCs, particularly the cancer stem cell (CSC) phenotype without affecting their "stemness"10. In the presence of M1 MФs, the majority of otherwise chemoresistant CSCs were responsive to carboplatin. Injection of M1 MФs into immune deficient mice harboring dormant BCCs reversed dormancy resulting in the $\mathrm{BCCs}$ becoming sensitive to carboplatin. The mice injected with M1 MФs showed prolonged survival with no evidence of the dormant BCC. In contrast, mice injected with $\mathrm{M} 2 \mathrm{a}$ MФs survived, but with persistence of the dormant BCCs. The data are discussed in the context of how BCCs may react to PRR stimulation, and the potential risk for long-term $\mathrm{BC}$ survivors.

\section{Materials and methods \\ Ethical statement}

The use of human blood and BM aspirates was approved by the Institutional Review Board (IRB) of Rutgers, Newark Campus. All subjects ranged were 18-35 years and signed the informed consent forms. The Institutional Animal Care and Use Committee (IACUC) approved the use of mice. The studies presented in this study adhered to the approved protocols.

\section{Culture of BM stroma/assessment of $M \Phi$ component}

Stroma was prepared from BM aspirates as described ${ }^{23}$. Total BM aspirate with nucleated cells $\left(10^{7}\right)$ were added to $25-\mathrm{cm}^{2}$ tissue culture flasks (Falcon 3109) in stromal media. At day 3, the red blood cells and neutrophils were removed by Ficoll-Hypaque density gradient and the mononuclear cells retrieved from each flask were readded in fresh stromal medium. The flasks were incubated until confluence with a weekly replacement of $50 \%$ fresh stromal medium.

Confluent stromal cells were assessed for MФ phenotype by immunofluorescence using anti-CD206 (M2) and anti-MHC-II (M1). MФs are trypsin resistant and deadherence requires treatment with $0.2 \%$ collagenase $^{24}$. The recovered MФs were incubated in Teflon jars for $2 \mathrm{~h}$ to allow any degraded membrane protein to be reexpressed. In five analyses with stroma from a different donor we observed $10 \pm 1.3 \%( \pm \mathrm{SD}) \mathrm{CD} 206+/ \mathrm{MHC}-\mathrm{II}-$ cells. Indeed, MФs, which are part of the stromal compartment, are important for functional hematopoiesis ${ }^{25}$.

\section{Monocyte/MФ culture}

The culture of M1 and M2a MФs was performed as described using human peripheral blood mononuclear cells (PBMCs) and BM mononuclear (BMNCs) ${ }^{26-28}$. Monocytes were selected by incubating the mononuclear cells with Dynabeads Flow Comp human CD14 ${ }^{+}$kit from Invitrogen (Carlsbad, CA). This resulted in cells that were positive for nonspecific esterase and $>99 \%$ CD14 + cells by flow cytometry (Fig. S1). The monocytes $\left(2 \times 10^{6}\right.$ cells $\left./ \mathrm{mL}\right)$ were incubated for $48 \mathrm{~h}$ in 6-well plates with RPMI 1640 containing $10 \% \mathrm{FCS}$ and $50 \mathrm{ng} / \mathrm{mL}$ of M-CSF ${ }^{29}$. The media were replaced with fresh media containing $10 \mathrm{ng} / \mathrm{mL}$ IFNy for M1 MФ and $20 \mathrm{ng} / \mathrm{mL}$ IL-4 for M2a MФ. MФs within $\mathrm{BM}$ stroma were ascribed the designation of $\mathrm{M} 2$ and were CD206+ and MHCII-.

\section{Cell synchronization}

BCCs were synchronized for $72 \mathrm{~h}$ in serum-free media supplemented with $1 \times$ insulin-transferrin-selenium. At synchronization, the cells were challenged with exosomes by replacing the media containing $10 \%$ FCS.

\section{Cell health}

The assessment of live/dead cells, referred as cell vitality, used two different dyes from Molecular Probes. The live cells can convert a redox dye (resazurin) into a fluorescent end product (C12-resorufin). SYTOX green dye is a permeable cell stain that is used to measure either injured or dead cells. Cells were incubated with $500 \mathrm{nM}$ C12-resazurin and $10 \mathrm{nM}$ SYTOX in $100 \mu \mathrm{l}$ $1 \times$ phosphate-buffered saline (PBS) for $15 \mathrm{~min}$ at $37^{\circ} \mathrm{C}$. Cell suspension was diluted with $400 \mu \mathrm{l} 1 \times$ PBS. Stained cells were immediately analyzed on FACSCalibur (excited at $488 \mathrm{~nm}$ and measured fluorescence emission at 530 and $575 \mathrm{~nm}$ ). Cell populations were gated into three groupslive, injured, and dead, and then quantified. 


\section{Exosome isolation and characterization}

Exosomes were isolated using a multi-step process. Large vesicles were eliminated by sequential centrifugation up to $50,000 \mathrm{~g}$ as described ${ }^{12}$. The remaining particles were pelleted by ultracentrifugation (Sorvall mTx 150, Thermo Scientific, Springfield, NJ) at $100,000 \mathrm{~g}$ for $18 \mathrm{~h}$. Vesicles expressing CD63 were immunoselected using CD63 magnetic bead isolation. The recovered particle size was verified by nanoparticle tracking analysis (NTA) using a NanoSight NS300 instrument (Amesbury, UK) as described $^{11}$. The data were analyzed with the NTA software (NANOSight version 2.3) using dilutions with deionized water. Three videos at a minimum of 200 completed tracks were collected at 30-s time intervals/ video per sample.

Exosomes were further distinguished by flow cytometry with magnetic beads coupled to anti-CD9-PE, anti-CD63FITC and anti-ALIX-APC (BD Biosciences).

\section{In vivo studies for roles of $M 1$ and $M 2$ MФs}

$\mathrm{BALB} / \mathrm{c}$ nude female mice (6 weeks) were purchased from Taconic Farms (Germantown, NY) and housed in an AAALAC-accredited facility. BC dormancy in BM was established by tail vein injection of Oct $4^{\text {hi }}$ MDA-MB-231 or T47D $\left(10^{3}\right.$ in $\left.0.2 \mathrm{~mL} 1 \times \mathrm{PBS}\right)$, as described ${ }^{10}$. To ensure dormancy mice were injected intraperitoneally with low dose of carboplatin $(2 \mathrm{mg} / \mathrm{kg})$ at days 2 and 5 , resulting in Oct $4{ }^{\text {hi }}$ BCCs in the endosteal regions of femurs. At day 7 , the mice were divided into groups of ten, and injected intravenously with (a) $10^{6}$ CMTMR-labeled M1 MФ in $0.2 \mathrm{ml} 1 \times$ PBS; (b) $10^{6}$ CMTMR-labeled M2a MФ in $0.2 \mathrm{~mL} 1 \times$ PBS; (c) Control, injected with $0.2 \mathrm{~mL} 1 \times$ PBS. Each experiment was repeated three times. At days 10, 12, and 15 , the mice were injected with $5 \mathrm{mg} / \mathrm{mL}$ carboplatin. At day 20, the mice were euthanized and the femurs and lungs were harvested. The femurs were washed to remove the cells and the endosteal region scraped and immediately analyzed for fluorescent cells. Femurs were also decalcified, embedded in paraffin and then sectioned at the digital imaging and histology core facility at New Jersey Medical School. Sections from the femurs of mice were subjected to immunohistochemistry for M1 and M2 MФs (see Supplemental method) In separate studies, survival curve was done for 30 days in three experiments, each with groups of 10 mice.

\section{Anti-miR-222 and -223 transfected MSCs}

MSCs were co-transfected with anti-miR-223 and antimiR-222 or negative control anti-miR using Lipofectamine RNAiMAX reagent (Life Technologies Invitrogen, Carlsbad, CA) as described ${ }^{11}$. The anti-miRs were purchased from Life Technologies-Applied Biosystems (Grand Island, NY). The MSCs were washed and then resuspended in PBS for injection into nude mice.

\section{In vivo studies for $М \Phi$ type between tumor-free and tumor-bearing mice}

$\mathrm{BC}$ dormancy was established in female $\mathrm{BALB} / \mathrm{c}$ nude mice (6 weeks) old as described, also explained above ${ }^{10}$. Another group was injected with PBS for the purpose of assessing $M \Phi$ type in femurs. The dormant group was further subdivided into two groups: Group 1 was injected $10^{6}$ MSCs transfected with anti-miR222/223 and Group 2, with control anti-miR. The anti-miRs can enter the dormant BCCs to reverse dormancy into proliferating $\mathrm{BCCs}^{11}$.

\section{Statistical analyses}

The data were analyzed using one-way ANOVA with Bonferroni's multiple comparison test as the post hoc test or two-way ANOVA with Bonferroni correction. A $p$ value $\leq 0.05$ was considered to be significant. The significant effects of exosomes and media were deduced by $p$ values of $<0.001$ in two-way ANOVA with Bonferroni post-test that compared cycling progression, G0/G1 vs. S-phase.

\section{Results \\ BM stromal M2 MФs to M1 type}

In order to include all components of BM stroma to understand $\mathrm{BC}$ dormancy, nonhematopoietic cells and MФs, stromal cultures are established with BM aspirates ${ }^{10,30,31}$. Western blots with whole cell extracts identified the marker of M2 MФs, mannose receptor C-type1 (MRC1/CD206), within stroma, similar to extracts from M2a MФ control (Fig. 1a) ${ }^{32}$. The normalized band densities showed CD206 significantly $(p<0.05)$ reduced in stroma as compared to control M2a MФs (Fig. 1b), consistent with $10 \%$ M2 MФs in stromal cultures, the literature and also, labeling of murine femur for CD206 $(11 \pm 2 \%, n=5)^{13,33}$.

Stromal M2 MФs could be switched to M1 phenotype as indicated by activation of TLR4 with LPS ${ }^{34,35}$ (Fig. 1c). Flow cytometry confirmed 10\% CD206+ cells in unstimulated stroma (Fig. 1d, left panel) and 10\% M1 MФs (MHC-II+) with LPS (Fig. 1d). The activated MФs expressed CD86 and reduced CD163 whereas unstimulated/vehicle $M \Phi$ s showed the reverse (Fig. 1e). Since CD163 is expressed on endogenous BM MФ, our model recapitulated occurrences in $\mathrm{BM}^{36}$. The switch of MФs to M1 type was confirmed M1 by cytokine production using purified MФs (Fig. 1h, i). Together the findings showed conversion of stromal M2 MФs into M1 type.

\section{Indirect polarization of $\mathrm{M} 2 \mathrm{a} M \Phi$ to $\mathrm{M} 1$ type via MSCs}

Since direct activation of TLR4 on M2 MФs switched to M1 type (Fig. 1a-e), we next asked if this can occur indirectly by MSCs ${ }^{11,37}$. M2a and M1 MФs from PBMCs or BMNCs of healthy donors expressed CD11b and CD86, 


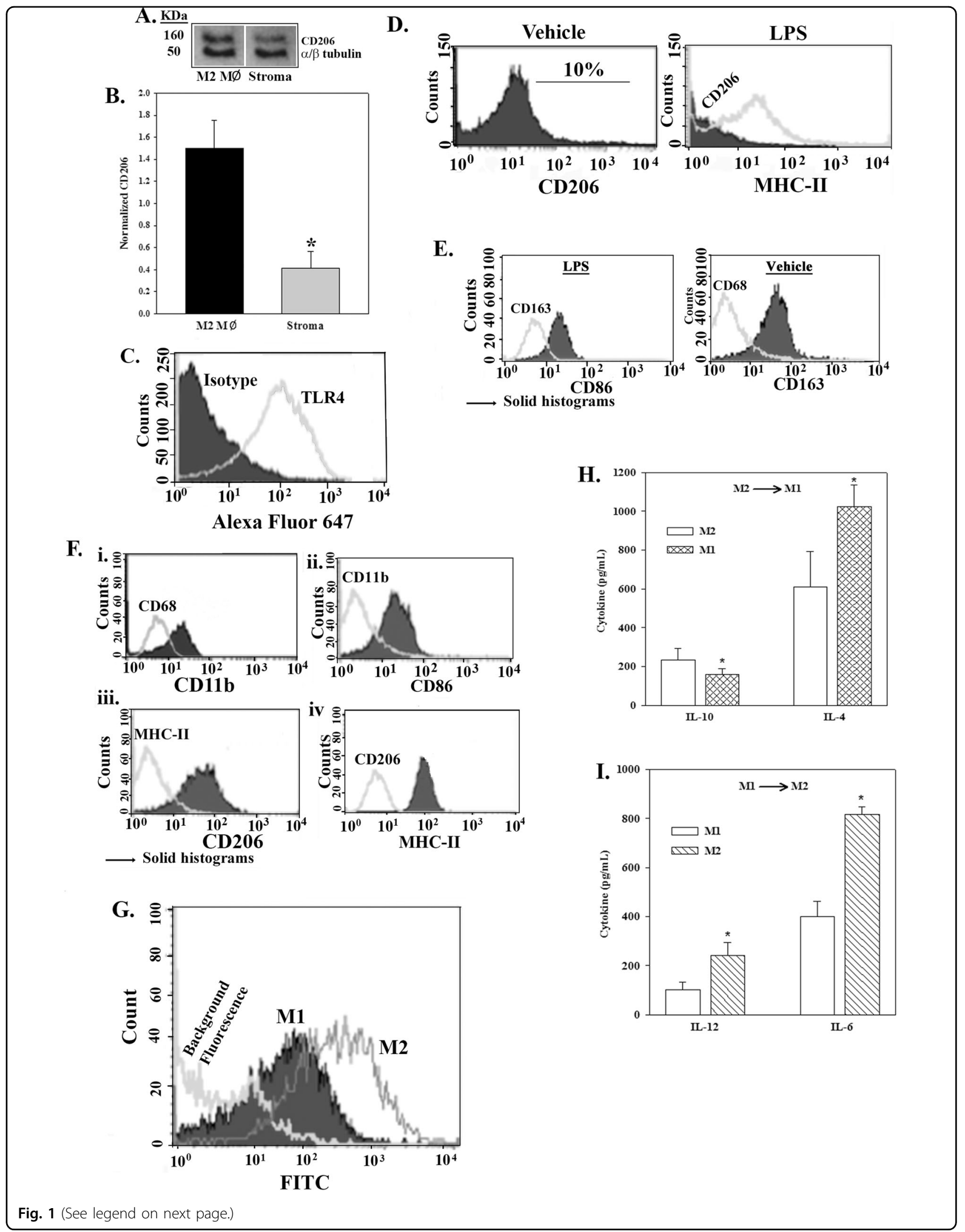


(see figure on previous page)

Fig. 1 Behavior of MФs within BM stroma. a Representative $(n=3)$ western blot for CD206 and a/b tubulin with whole cell extracts from three different donors of stroma or purified M2a MФ. $\mathbf{b}$ The band densities for CD206 were normalized using the bands for a/b tubulin. The results are presented as the mean $\pm \mathrm{SD}, n=3$. c Representative $(n=3)$ flow cytometry for TLR4 with BM stroma stimulated with $10 \mathrm{ng} / \mathrm{mL}$ LPS. $\mathbf{d}$ Representative $(n=3)$ histogram for CD206 and MHC-II with stromal cells stimulated with vehicle or $10 \mathrm{ng} / \mathrm{mL}$ LPS, respectively. The right panel shows the overlay of CD206 and MHC-II. e Stromal cells $(n=3)$ were stimulated with $10 \mathrm{ng} / \mathrm{mL}$ LPS or vehicle. The cells were removed with $0.2 \%$ collagenase followed by a 2-h incubation to allow cell surface proteins to recover. The CD14+ cells were gated by flow cytometry and then analyzed for CD68 and CD163. The histograms are shown as overlays with the solid graphs representing the label on the $x$-axis. $\mathbf{f}$ PBMC and BMMC-derived M2a and M1 MФs were studied for CD11b and CD68. (i-ii) The histogram were overlaid and the solid graphs are indicated by the $y$-axis. The M2a MФs were treated with vehicle (iii) or LPS-stimulated MSCs (iv). After $16 \mathrm{~h}$, the cells were labeled for CD206 and MHC-Il and then analyzed by flow cytometry. The histograms for each were overlaid and the markers shown in the solid graphs are placed on the $y$-axis. $\mathbf{g ~ M 1}$ and M2 MФ were studied for phagocytic properties by incubating with IgG-FITC coupled to beads. The cells were washed and then analyzed by flow cytometry for FITC. Shown is a representative overlay of three independent experiments. $\mathbf{h}$ MDs in cultures with activated MSCs were negatively selected and then studied for IL-10 and IL-4 levels, $\pm \mathrm{SD}(\mathrm{pg} / \mathrm{mL}), n=4$. I M1 MФs from " $\mathrm{H}$ " were retained for $24 \mathrm{~h}$ to re-repolarize to M2a MФ and then the media analyzed for IL-12 and IL-6. The results are expressed as the mean levels $(\mathrm{pg} / \mathrm{mL}) \pm \mathrm{SD}$

respectively (Fig. 1f, i-ii). LPS- (stimulated) or vehicle(unstimulated) MSCs, added to M2a MФs for $16 \mathrm{~h}$, indicated no change in $M \Phi$ phenotype by unstimulated MSCs (CD206+) whereas stimulated MSCs switched the MФs to M1 phenotype (MHC-II+) (Fig. 1f, iii-iv). The MSCs did not affect the $M \Phi$ function, based on phagocytosis of fluorescence latex particles (Fig. 1g). The switch to M1 MФs were validated by higher IL-4 level as compared to M2a MФs (Fig. 1h). In summary, this section showed indirect polarization of M1 and M2a MФs by MSCs. The mechanisms by which MSCs control MФ types is included in a separate publication.

\section{Cytokine production by $\mathrm{M} 1$ and $\mathrm{M} 2 \mathrm{M} \Phi \mathrm{s}$}

In order to validate the flow cytometry analyses in M2-M1 MФ conversion, we performed ELISA for associated cytokines $^{38}$. M2 to M1 MФ conversion caused a significant $(p<0.05)$ decrease of IL-10 whereas M1 to M2 MФ increased IL-6 and IL-12 (Fig. 1h, i). The results indicated functional plasticity by M1 and M2 MФs, consistent with the literature ${ }^{38}$.

\section{Discerning $М Ф$ type in tumor and nontumor bearing femurs}

This set of studies validated the predominance of M2 MФs in stromal cultures (Fig. 1). We first analyzed the femurs of nude female $\mathrm{BALB} / \mathrm{c}$ mice by immunohistochemistry for M1 MФs (MHC-II) and M2 MФs (CD206). Since the nude mice are deficient in T-cells and the mice were not activated immunologically, the MHC-II could be a surrogate of M1 MФ. We did not detect MHCII but CD206 was detected, verifying M2 MФs in mice femur (Fig. 2a).

Next, we studied the BALB/c mice femurs for $М \Phi$ type using models with dormant BCCs and reverse dormancy when the mice was treated with anti-miR222/223 ${ }^{11}$ (Fig. 2b). M1 MФs were detected during reverse dormancy (Fig. 2c, left panel) whereas those with dormant BCCs $(\mathrm{GFP}+)$ showed cells positive for CD206 (blue)
(Fig. 2c. right panel). In summary, M2 MФs were present in nude BALB/c femurs with dormant BCCs and M1 type during reverse dormancy. We estimated about 5-10\% M2 MФs in the femurs, close to the endosteal region.

\section{Relative efficiencies in GJC by M1 or M2 MФs with CSCs}

We reported on sustained BC dormancy by GJIC between $\mathrm{BM}$ stroma and $\mathrm{CSCs}^{10}$. In order to determine if M2 MФs can contribute to GJIC, we negatively select M1 and M2 MФs within BM stroma and then analyzed the extracts for Cx26, Cx32, and Cx43 by western blots. Band densities for Cx32 and Cx43 were significantly $(p<0.05)$ higher for M2 MФs and similar for Cx26 (Fig. 3a, b).

The function of Cxs were studied in a two-way dye transfer assay between MФs and BCC subsets in the presence or absence of a pharmacological agent (1-octanol) (Fig. 3c, top panel). The CSCs were green due to pOct4a-GFP and were labeled with CFDA (green). MФs were labeled with CMTMR (red). Flow cytometry for CMTMR transfer in the GFP cells (CSCs) indicated transfer from M2 MФ to CSCs, which was significantly reduced with M1 MФs (Fig. 3c). The dye transfer was specific based on a blunting effect by 1-Octanol, which retained cell viability (Fig. $3 \mathrm{~d}$ and S2).

\section{Distinct effects by $M 1$ and $M 2 a M \Phi$ on BCC cycling}

Since M1 MФs showed less efficiency in GJIC with CSCs, we asked if M1 MФs can reverse CSC quiescence. Equal numbers of unsorted BCCs and M1 or M2a MФs (from BMNCs), co-cultured for $72 \mathrm{~h}$, showed significantly $(p<0.05)$ increased BCC proliferation as compared to M2a MФs (Fig. 3a). Gating of the CSC subset (bright GFP $)^{10}$, we noted a significant $(p<0.05)$ decrease in G1 phase and increase in S/G2 phases by M1 MФs (Fig. 4b and Fig. S3). The effects were specific for MФs since the major stromal cells (fibroblasts) did not affect the cycling of BCCs (Fig. S4).

Limiting dilution of tumorspheres from CSCs (Oct4 high) in 72-h co-culture with M1 or M2 MФs indicated no effect 

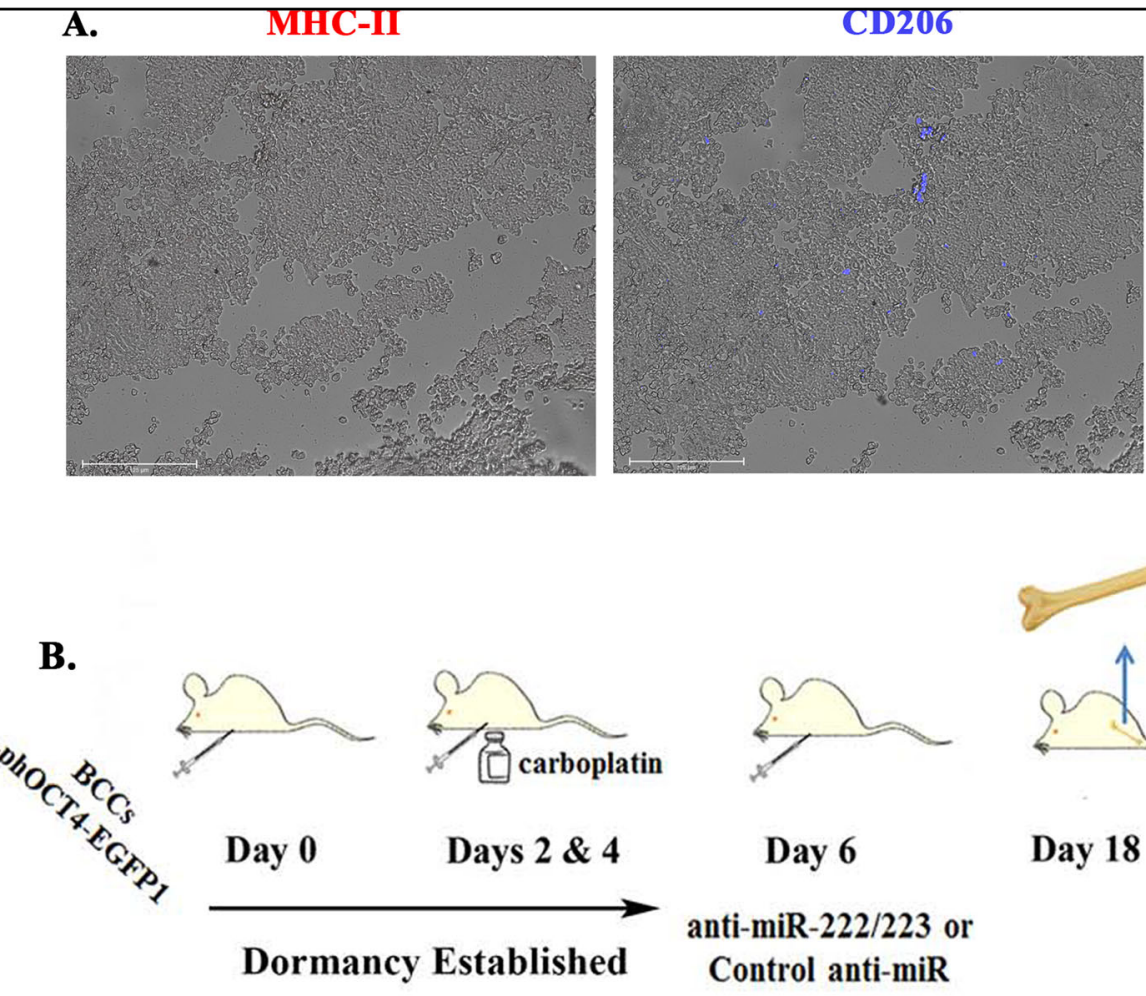

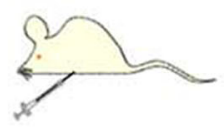

Day 6
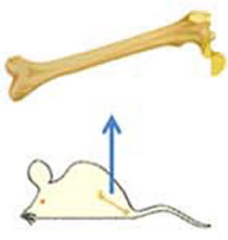

\section{Day 18}

anti-miR-222/223 or

Control anti-miR
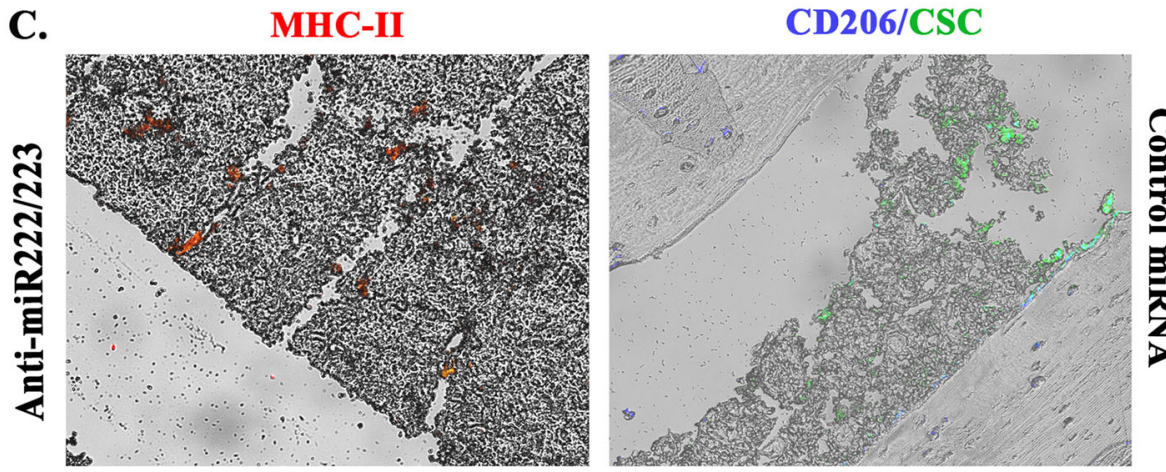

Fig. 2 MФ type in BALB/c femurs and with dormant and reverse dormant BCCs. a Femurs were subjected to immunohistochemistry for MHC-I using indirect labeling with secondary Texas Red IgG (left panel) and for CD206 with Alexa Fluor conjugated secondary lgG (right panel). b Dormant BCCs were established in female BALB/c. Dormancy was assured by injected the mice with $5 \mathrm{mg} / \mathrm{kg}$ of carboplatin at Days 2 and 4 . At Day 6 , the mice were injected with MSCs transfected with anti-miR222/223 previously shown to reverse dormancy. At day 18, the mice were euthanized and the femurs harvested. c Sections from the femurs in "B" were analyzed for MHC-II and CD206. The green fluorescence cells in the right panel represents CSCs. The images represents five independent sections, each from a different mouse

on self-renewal after fiver serial passages. Representative spheres are shown from passage 5 (Fig. 4b, lower panels).

\section{Effects of M1 and M2 MФs on the cycling of BCC subsets}

The effect of MФs on cell cycle of distinct BCCs subsets was assessed with Oct4A-GFP transfectants ${ }^{10}$ (Fig. 4c, left panel). The low-DNA/non-cycling cells (7-AAD) were analyzed for low RNA (Pyronin $Y$ ) to discern $G_{0}$ and $G_{1}$ phases. The CSCs $\left(\mathrm{GFP}^{\mathrm{hi}}\right)$ were mostly in $\mathrm{G}_{0}$ phase with M2a MФs (Fig. 4c, middle and top panel, 15.7\%). M1 MФs significantly $(p<0.05)$ reduced the percentage of $\mathrm{G}_{0}$ cells (Fig. 4c, right and top panel, 8.8\%). The other subsets were not affected regardless by the MФ type (Fig. 4c, lower two panels). In summary, the results indicated that the CSCs were mostly affected by M1 and M2 MФs with respect to their cycling phase.

\section{Effects of MФ-derived exosomes on the cycling phase of BCCs}

Figure $5 \mathrm{a}$ outlined the approach that was used to analyze the role of $\mathrm{M} \Phi$ secretome on $\mathrm{BCC}$ cycling phase. Stroma was activated with $10 \mathrm{ng} / \mathrm{mL}$ LPS (TLR4), 

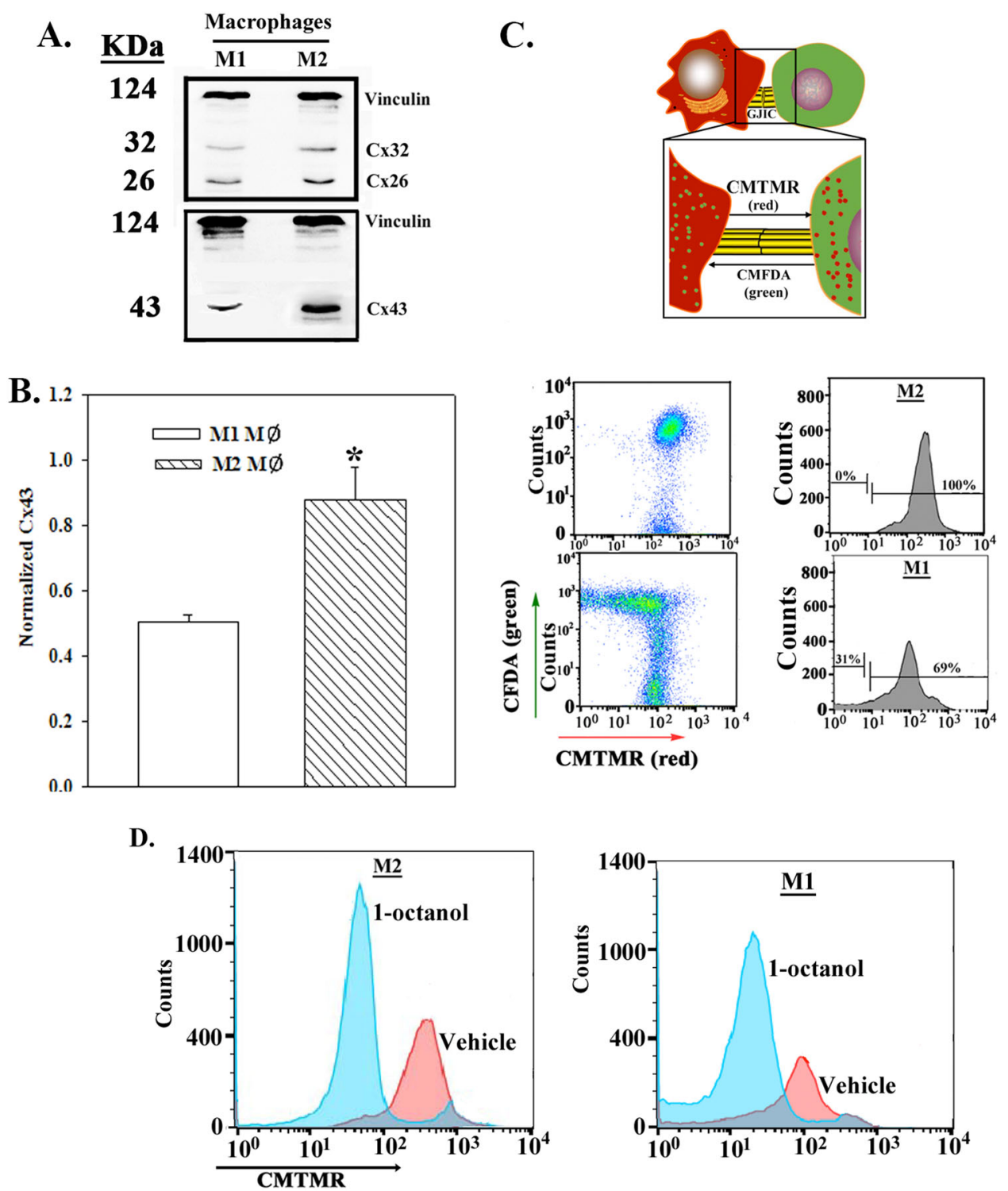

Fig. 3 GJIC between CSCs and M1/M2a MФs. a Western blot for Cx26 and Cx32 (top panel) and Cx43 (bottom panel) with extracts from BMNCderived MDs. $\mathbf{b}$ The densities for the top bands (total $\mathrm{C} \times 43$ ) were normalized with bands for Vinculin and then presented as the mean $\pm \mathrm{SD}, n=3$. ${ }^{*} p<$ 0.05 vs. M1 MD. c Top panels show the experimental design in which the CSCs were green due to Oct4a-GFP. The CSCs were labeled with CFDA (green) and the MФs with CMTMR (red). The M2 MФs (middle panels) or M1 MФs (third panels) were co-cultured at 1:1 ratio with $10^{6}$ CSCs from MDA-MB-231 or T47D in 6-well plates. After $24 \mathrm{~h}$, the CSCs were selected by trypsin digestion and then examined for CMTMR transfer by flow cytometry. Representative histograms and scatter plots are shown for MDA-MB-231. d The studies in " $\mathrm{C}$ " were repeated in the presence of $300 \mu \mathrm{M}$ 1-octanol or vehicle

$10 \mathrm{ng} / \mathrm{mL}$ Poly-IC (TLR3) or vehicle, and after $24 \mathrm{~h}$ the $M \Phi$ s were negatively selected by trypsin. The stimulated MФs expressed MHC-II (M1) and unstimulated, MHC-II-/CD206+ (M2). The purified MФs were reincubated in exosome-free media. After $24 \mathrm{~h}$, the media were collected and then fractionated into exosome and exosome-cleared media. Exosomes $\left(4 \times 10^{7}\right.$, per dose-response, Fig. S5) and the cleared media were added to $10^{6}$ serum-starved BCCs, which were mostly in $\mathrm{G}_{0} / \mathrm{G}_{1}$ phase and good health (Fig. $5 \mathrm{~b}, \mathrm{c}$ ). The exosomes were $<120 \mathrm{~nm}$ and expressed tetraspanin proteins (Fig. 5d, e).

The cycling phase of nontumorigenic HBL-100 cells were not influenced by the exosomes or cleared media (Fig. 5f). However, exosomes from activated MФs (M1) reduced the cycling quiescence of BCCs (Fig. 5f, left panels). This effect was specific to exosomes since $10 \%$ FCS showed no change (Fig. 5f, right panels). The results indicated that exosomes from activated MФ excited BCCs from cycling quiescence. 

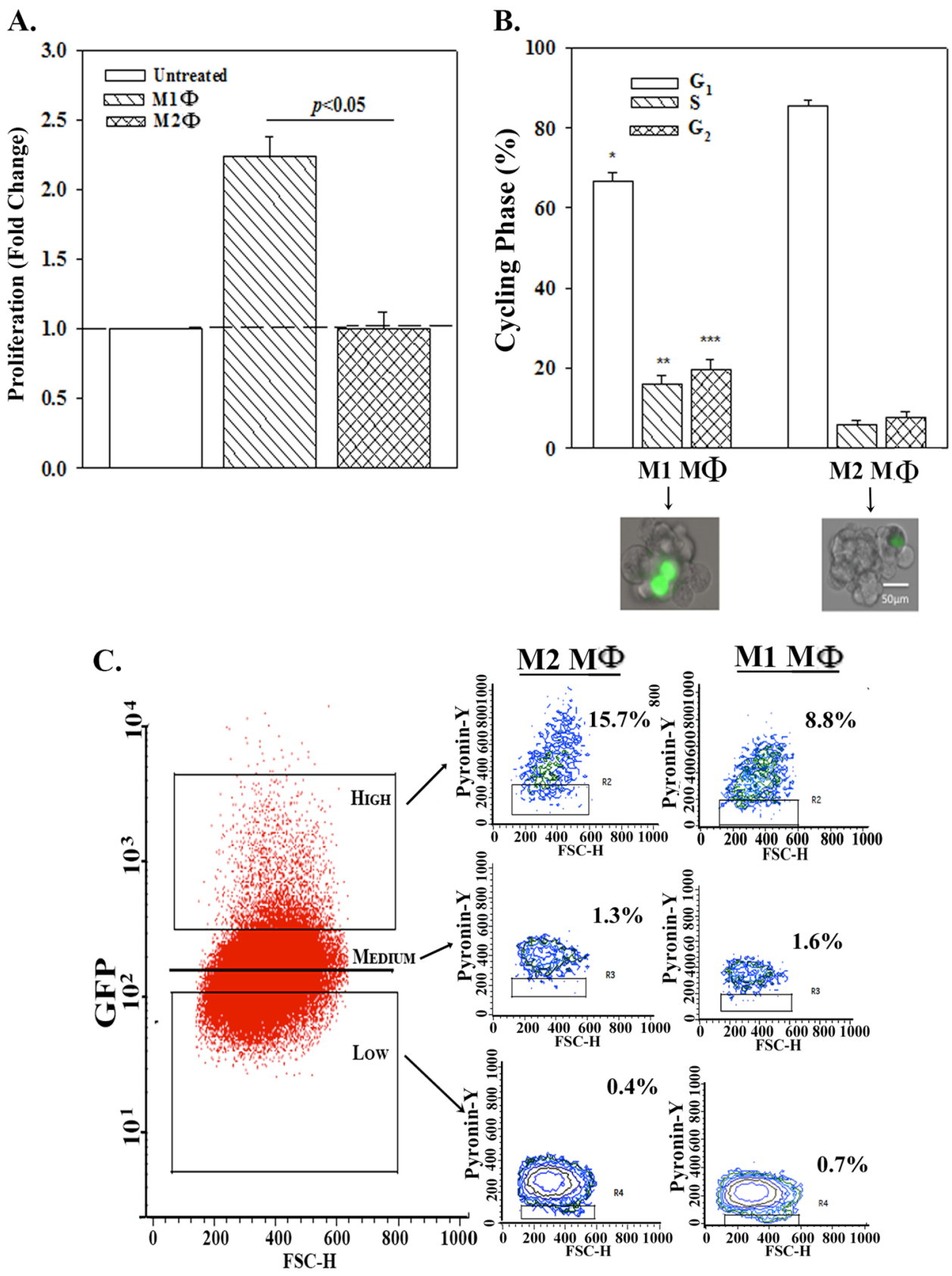

Fig. 4 Increased proliferation and cell cycling of BCCs by M1 MФ. a BCCs were co-cultured with M1 or M2 MФs at 1:1 ratio or alone at $10^{6}$ cells each in 775 tissue culture flasks. After $72 \mathrm{~h}$, cell proliferation was assessed by Cyquant Assay. The data with BCCs alone are normalized to 1 and the experimental expressed as fold change $( \pm S D, n=4)$. b The studies in " $A$ " were repeated with BCCs stably transfected with pOct4a-GFP. After $72 \mathrm{~h}$, the BCCs were analyzed for cell cycle by PI labeling. The data for cells in G1, S, and G2 phases are presented as the mean \pm SD, $n=4$. Tumorspheres from the pOct4a-GFP BCCs in cultures with MФs were cultured to form spheres by limiting dilution. Images at 200x magnification with the Evos $f$ imager are shown below. $\mathbf{c}$ The studies in "B" were repeated except the cycling analyses were done with 7-AAD and Pyronin Y labeling. Left panels show the gating scheme for the analyses based on relative GFP intensities. The cells with low-DNA content were further analyzed for low RNA to represent cells

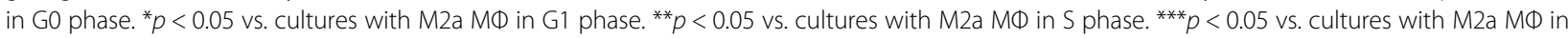
G2 phase

\section{MФ-derived exosomes in BCC migration}

We determined if M1-mediated cycling transition of CSCs correlates with migration and epithelial/mesenchymal transition (EMT)/MET. Migration using scratch assay with CSCs, mostly in $G_{0} / G_{1}$ cycling phase ${ }^{10}$ and, treated with $2 \times 10^{7}$ exosomes from MФs (M2 or M1) or without 
A.

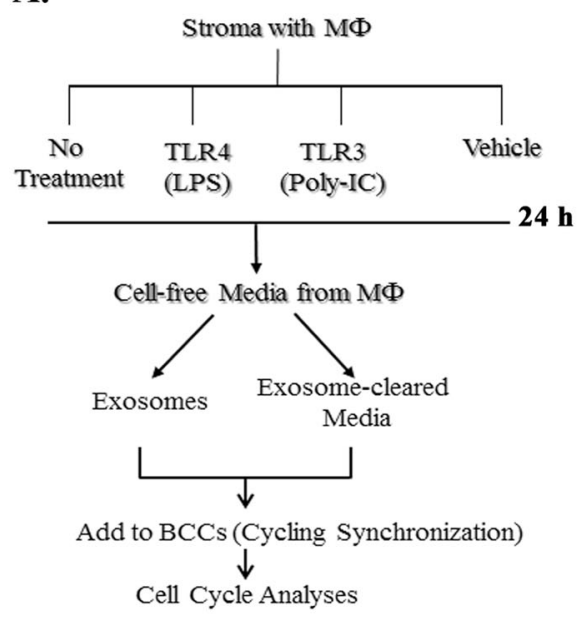

C.

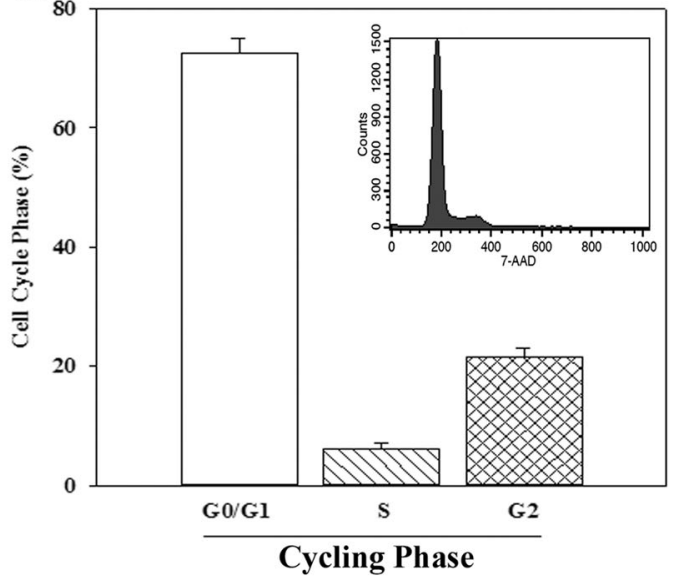

D.

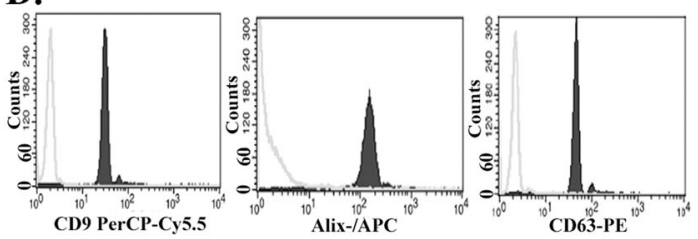

E.

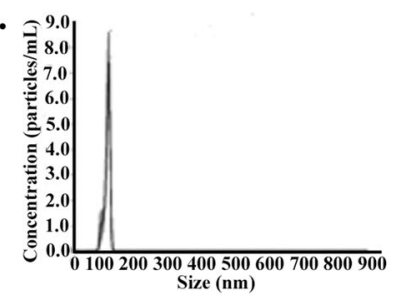

B.

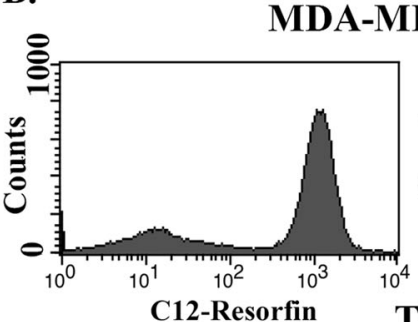

T47D
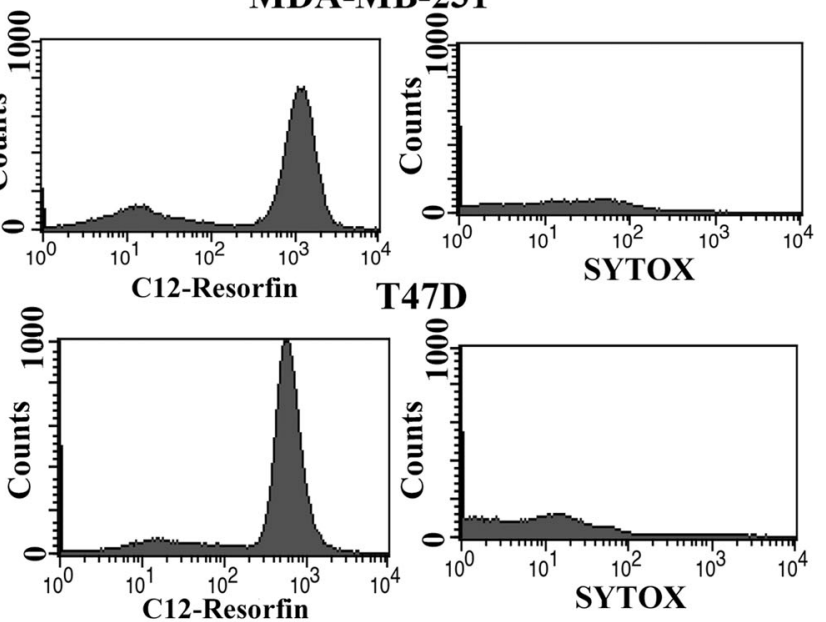

F.
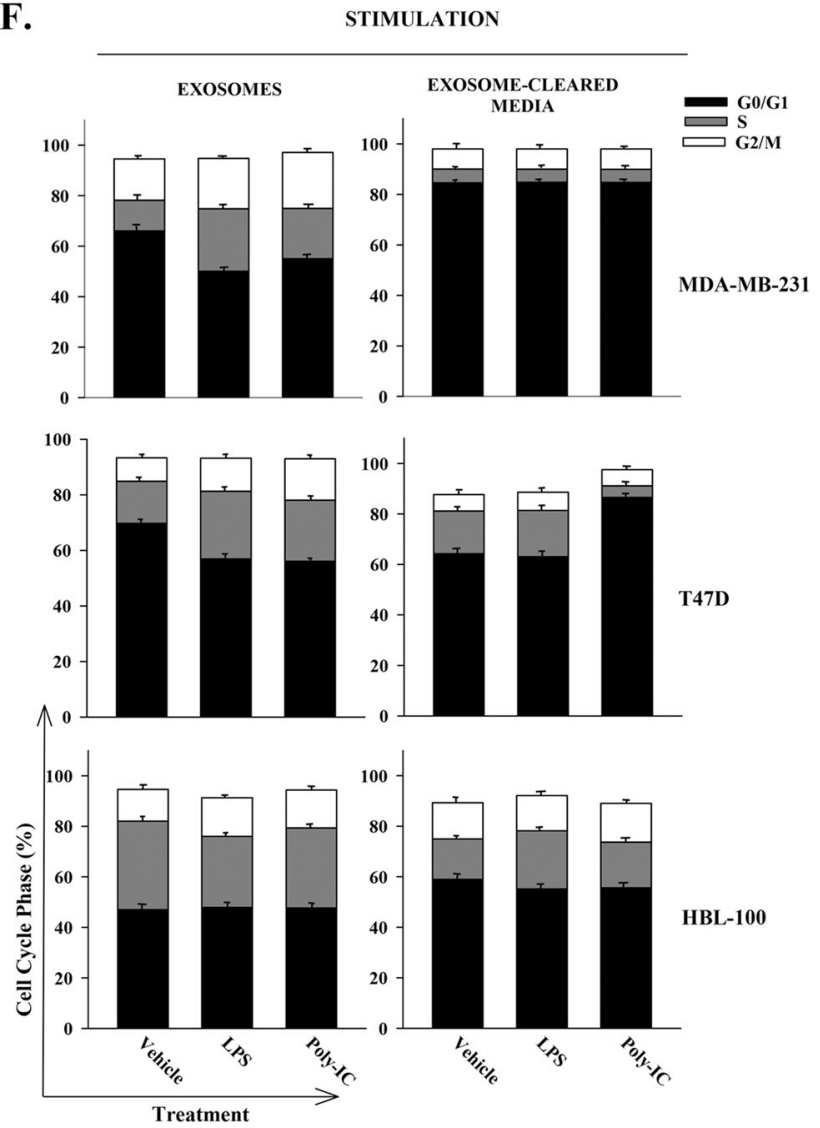

Fig. 5 Exosomes from MФs in the behavior of quiescent BCCs. a The diagram shows the overall experimental design used for the data in this figure. $\mathbf{b}$ The serum-starved/synchronized BCCs were assessed for cell health as described in the Materials and Method section. $\mathbf{c}$ Cell cycle analyses were performed with the serum-starved BCCs. Inset shows a representative histogram of results of MDA-MB-231 with PI labeling. The results are shown for percent cells in different cycling phase, mean $\pm S D, n=6$. d A representative histogram of flow cytometry for tetraspanin proteins for the isolated exosomes. e A representative graph of the NTA is shown for the isolated exosomes. $\mathbf{f}$ Stacked bar graphs of cell cycle analyses by PI labeling of serum-starved BCCs treated for $24 \mathrm{~h}$ with MDs-derived exosomes. The MDs were isolated from stroma and then treated with vehicle, $10 \mathrm{ng} / \mathrm{mL} \mathrm{LPS}$ and $10 \mathrm{ng} / \mathrm{mL}$ poly-IC as outlined in "A". The results are mean\% cells in cycling phase, $\pm \mathrm{SD}, n=7$ 


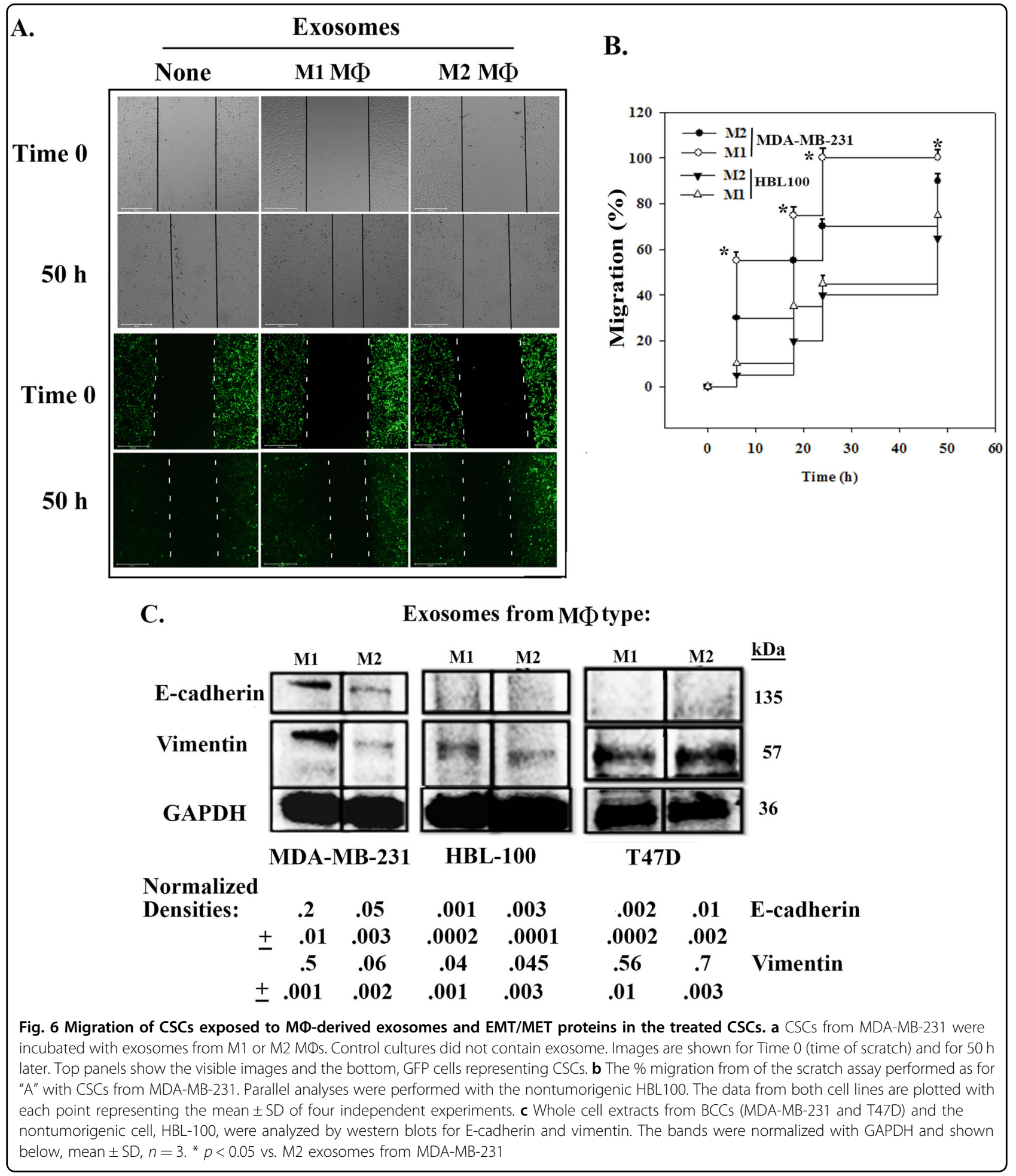

exosomes. A representative image for MDA-MB-231 is shown for time 0 up to $50 \mathrm{~h}$ (Fig. 6a). The plot (mean \pm SD, $n=4)$ of the scratch assay indicated a significant $(p<0.05)$ increase in migration with exosomes from M1 as compared to M2 (Fig. 6b).
Western blots for vimentin and E-cadherin were increased in MDA-MB-231 exposed to exosomes from M1 and M2 MФs. This suggested mixed behavior of BCCs with respect to EMT and MET (Fig. 6c). There was no marked change for T47D and HBL-100 cells. 


\section{Exosomes from M1 MФs enhanced BCC cycling via NFKB}

Active NFKB can regulate cell proliferation, survival, and tumor progression ${ }^{39-41}$. We asked if M1-derived exosomes can induce BCC cycling via active NFKB. Functional studies were needed because phosphorylated (phospho) p65 in the cytosol may not be active ${ }^{42}$. Serumstarved BCCs and nontumorigenic HBL-100 cells were treated with exosomes from M1 or M2 MФs. Flow cytometry showed increase of p65 in all cell lines but only M1 MФ-derived exosomes induced TLR4 on the BCCs ( 98\% vs. $<4 \%$ on HBL-100) (Fig. 7a), suggesting that increased TLR4 was endogenously induced and not transferred by exosomes. The data shown as average mean fluorescence intensity (MFI) verified p65 increase in cells treated with M1-derived exosomes (Fig. 7b). To further verify $\mathrm{NF}_{\mathrm{K}} \mathrm{B}$ activation, we analyzed the cell extracts for phospho-p65 and showed increases in band intensities when the BCCs were treated with M1-derived exosomes (Fig. 7c).

The role of p65 in cell cycle behavior of BCCs was studied by pretreating the synchronized cells with $20 \mu \mathrm{M}$ of NFKB inhibitor (pyrrolidine dithiocarbamate, PDTC) for $24 \mathrm{~h}^{43}$. Exosomes from M1 or M2 MФs were added and $24 \mathrm{~h}$ later, the cells were analyzed for cycling phase by PI labeling. PDTC only blunted cycling of M1-derived exosomes for MDA-MB-231 (Fig. 7d), suggesting a different mechanism for low-invasive/ metastatic T47D.

\section{MФ-derived exosomes on carboplatin sensitivity}

Since M1 MФs can transition quiescent BCCs to cycling cells, we asked if the secreted exosomes can sensitize BCCs to carboplatin. This drug allows us to treat all BCCs, irrespective of the hormone status. CSCs, which are in $G_{0} / G_{1}$ phase, were incubated with $4 \times 10^{7}$ exosomes from M2a or M1 MФ, followed by treatment with $50 \mu \mathrm{g} /$ $\mathrm{mL}$ of carboplatin. After $72 \mathrm{~h}$, flow cytometry for the CSC health indicated significant $(\sim 80 \%, p<0.01)$ cell death by M1 MФs (Fig. 8a). We asked if the resistant $20 \%$ CSCs (Fig. 8a) could be explained by P-gp, which is the protein for the multidrug resistance (MDR1)/ATP binding cassette $^{10}$. Flow cytometry indicated similar MFIs for P-gp in CSCs, exposed to M1 exosomes followed by treatment with vehicle and carboplatin (Fig. 8b). These findings suggested that P-gp expression was dependent on the exosomes and not carboplatin.

\section{Chemosensitivity of M1 MФs}

Dormant BCCs are carboplatin resistant ${ }^{10}$. Since M1 MФs caused quiescent BCCs to cycle, we asked if M1 MФs can be combined with carboplatin to treat dormant BCCs. Using the approach in Fig. 8c, we established dormancy in nude BALB/c and then injected CMTMR (red)-labeled M1 and M2 MФs ${ }^{10}$. Dormancy was ensured with low carboplatin dose up to day 5 . At day 7 , the MФs were injected and 3 days later, mice were treated with higher doses of carboplatin.

Prior to carboplatin treatment, 3 days MФs injection, we observed no structural change in decalcified hematoxylin-stained femurs (Fig. 8d, top). We also observed migration of CMTMR-labeled MФ to the femur (red) within the cellular regions (DAPI, blue) (Fig. 8d, row 2-enlarged sections below).

Prior to carboplatin treatment, we observed CSCs (green) in the cellular region of lungs (DAPI, blue) in mice treated with M1 MФs but undetectable CSCs with M2 injection (Fig. 8e). The latter observation indicated that CSCs within the lungs could not be explained by trapped cells.

At 20 days after the last treatment, we examined scraped sections of femurs with M1 MФs, treated or untreated with carboplatin. Only red fluorescent cells were noted with carboplatin, indicating elimination of CSCs with Oct4A-GFP (Fig. 8f, left). In contrast, untreated mice showed yellow regions, indicating GFP (injected CSCs) and red CMTMR (MФs) (Fig. 8f, right). These results indicated that the M1 MФs enhanced the response of CSCs to carboplatin.

Survival studies ( $n=10$ /group) for mice injected with M1 MФs without carboplatin succumbed by day 22 (Fig. 8g, open circle). The mice with M1 MФs or M2 MФs treated with carboplatin survived up to 30 days (Fig. 8g, solid circle and triangle). M2 MФs maintained the CSCs in dormancy, hence the enhanced survival. Scraped sections of the endosteal region showed $\mathrm{GFP}(+)$ cells (injected CSCs) as compared to undetectable CSCs in mice with M1 MФs and carboplatin (Fig. 8h). In summary, M1 MФs caused the dormant CSCs to become sensitive to carboplatin whereas M2 MФs retained dormancy.

\section{Discussion}

This report supports insights on how components of BM stroma can support the clinical and experimental evidence on disseminated BCCs adapting dormancy ${ }^{44}$. BM stroma facilitates dormancy partly through GJIC, miRNA, exosomes, and cytokine regulation ${ }^{12,45}$. This study demonstrates sustained dormancy by M2 MФs, which could be reversed by M1 MФs.

Unactivated BM stroma contains M2 MФs as demonstrated in stromal cultures and, mouse femurs (Figs. 1 and 2 ). Since inflammation can cause hematopoietic suppression, it is expected that stroma will contain antiinflammatory M2 MФs ${ }^{13}$. Our model recapitulated occurrences within BM as CD163+ MФs are detected in the stromal cultures (Fig. 1) ${ }^{36}$. Similar to GJIC between CSCs and BM stromal cells, we also showed a similar results for $\mathrm{M} 2 \mathrm{M \Phi s}^{10,12}$ (Fig. 3). GJIC was significantly reduced when M2 MФs were switched to M1 (Fig. 3). M1 


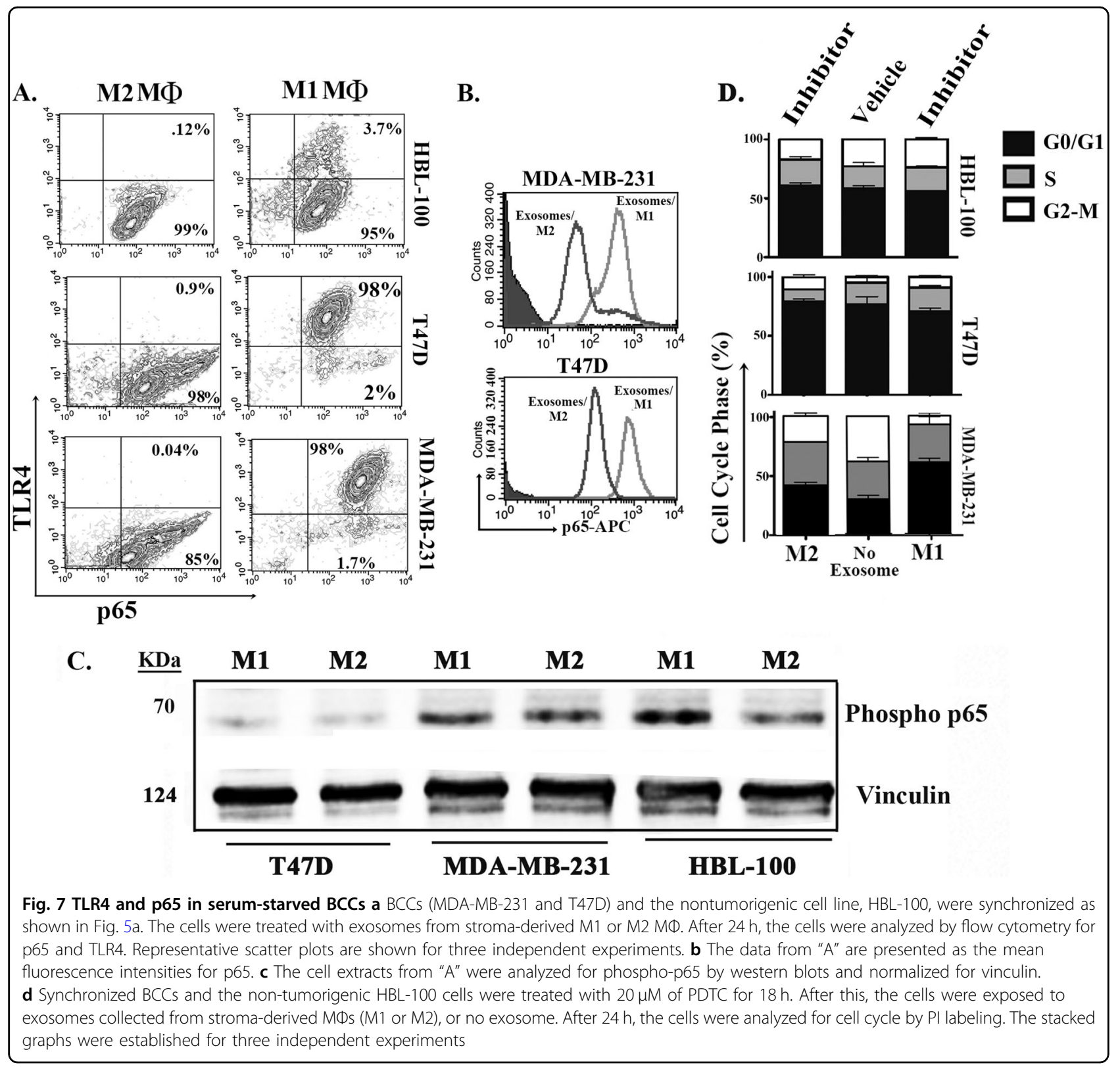

exosomes enhanced CSC migration and also affected the MET/EMT program (Fig. 6). The latter findings are in line with the literature that reported on a dynamic switch between EMT and MET during migration ${ }^{46}$. It is possible that $\mathrm{M} 1 \mathrm{M} \Phi$ s might revert to $\mathrm{M} 2$ and this could result in exosomes from both МФ types. Regardless, we deduced a complex effect of $\mathrm{M} \Phi$-derived cytokines on quiescent BCCs. These findings are consistent with other studies showing a role for M1 and M2 MФs in the behavior of low-invasive MCF7 cells ${ }^{47}$. The data also supported an indirect role for MSCs in determining the fate of MФs (Fig. 1f-i).

M1-mediated enhancement of CSC cycling was due to exosomes since the vesicle-free media did not show the same effect (Figs. 4 and 5). Going forward, roles for cytokines need to be examined since there is a potential for technical errors such as degradation of labile cytokines. Also, if there are cytokine receptors on the exosomes, this might absorb the soluble cytokines. A timeline study will account for cytokines with different peak times for rapid autocrine binding to the cognate receptors.

Our findings on M1-derived exosomes to partly explain reversed dormancy are interesting. Although not tested, we speculate that exosomes with different molecular contents from unstimulated MSCs would be important to maintain GJIC between MФs and stroma. In unpublished studies we have found that CSCs release exosomes that enter stromal cells. The stroma responded by secreting 


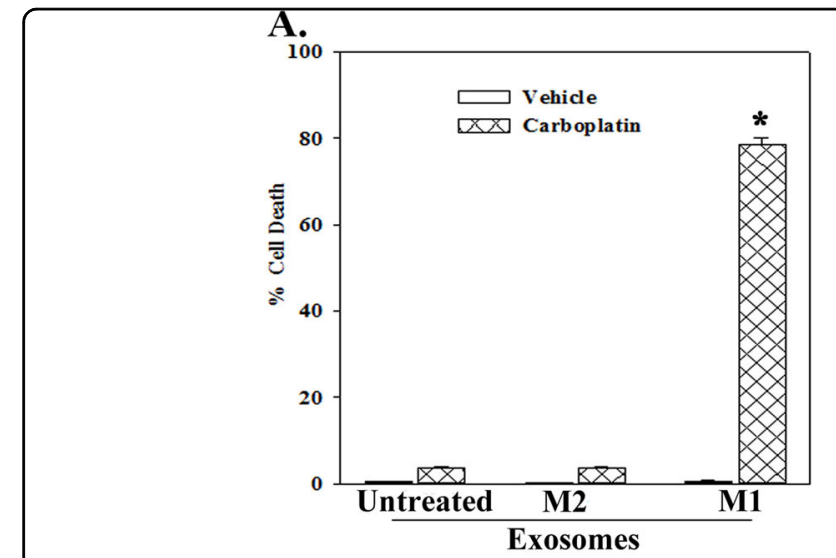

B.

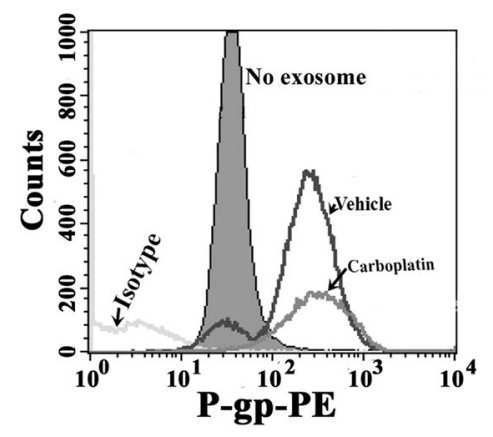

C.

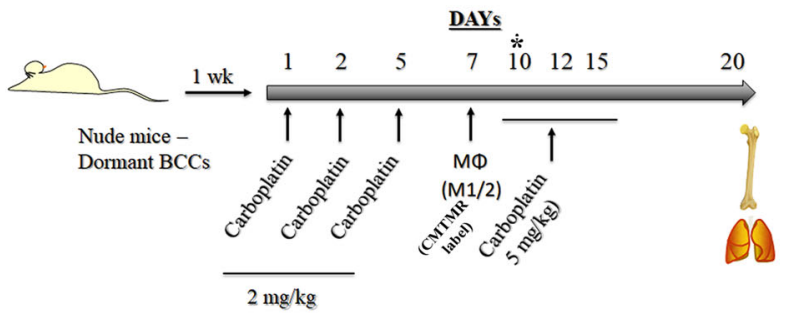

D.

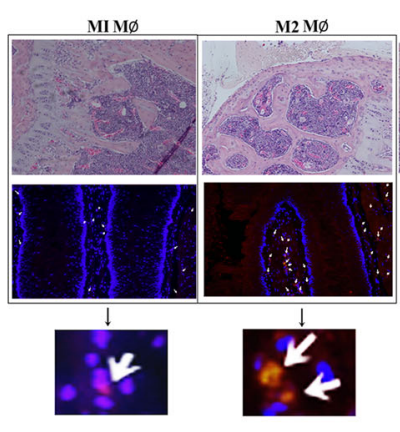

E.

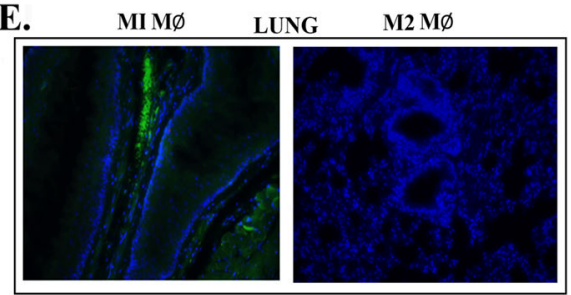

H.

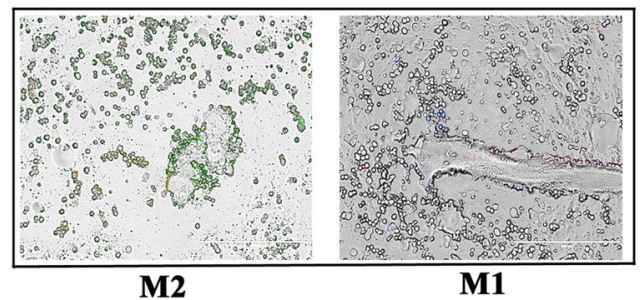

F.

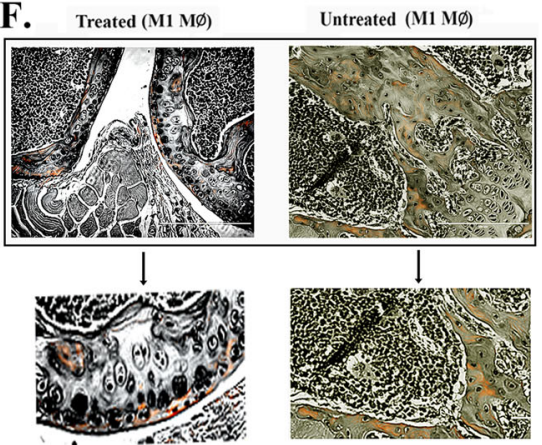

$\underline{\text { Enlarge insets }}$

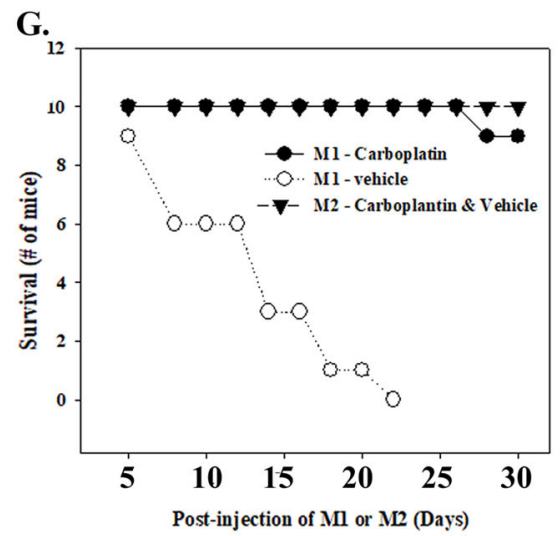

Fig. 8 (See legend on next page.) 
(see figure on previous page)

Fig. 8 Influence of M1 and M2 MФs on the sensitivity of dormant BCCs to carboplatin. a CSCs were cultured with exosomes from stromaderived M1 or M2 MDs or without exosomes. After $24 \mathrm{~h}$, the cells were treated with $50 \mu \mathrm{g} / \mathrm{mL}$ carboplatin or vehicle for $72 \mathrm{~h}$ and then studied for cell health. The \% cell death is presented as the mean \pm SD, $n=4 .{ }^{*} p<0.01$ vs. carboplatin-treated cells with M2 exosomes or untreated. $\mathbf{b}$ The CSCs treated with exosomes from "A" were studied for P-gp by flow cytometry. c A diagram outlining the method used in the in vivo studies with nude female BALB/C mice. The asterisk represents the time when three mice were euthanized to determine if the injected CMTMR-labeled MDs entered the femurs. $\mathbf{d}$ Hematoxylin-stained femurs of mice with dormant CSCs, injected with M1 or M2 MФs, or no MФ (top panels). The middle panels shows cellular regions labeled with DAPI (blue) and red fluorescent MФs. The lower panels show a large inset of what the arrows represent in the middle panels. e The lungs of mice were examined at day 10 for CSCs (GFP+). Shown are sections labeled with DAPI (blue) and the GFP cells shown in mice injected with M1 MФs. $\mathbf{f}$ Sections of decalcified femurs of mice injected with M1 MФs with or without carboplatin treatment. Shown are only red regions for the treated femurs indicating labeled MФs. The untreated femurs have yellow (MФs and CSCs), red (MФs), and green (CSCs) sections. Below are enlarged insets of the sections. g A survival curve for mice (10/group) injected with M1 or M2 MФs followed by treatment with caroboplatin or vehicle. Since the survival of treated mice injected with M2 MФs were similar to untreated mice as well as mice without MФs but given carboplatin, the values were plotted together. $\mathbf{h}$ Scrapings of the endosteal regions of mice femurs for GFP cells (CSCs) from those injected with M2 (left) and M1 (right) MФs

cytokines to support GJIC with the CSCs, hence a method of sustained dormancy. In other unpublished findings, we have observed dedifferentiation of BCCs into cells with stem cell phenotype following exposure of exosomes from BM microenvironmental cells. Thus, the latter findings link exosomes with GIIC since the CSCs show higher efficiency of forming GIIC with other cells in the $\mathrm{BM}$ microenvironment ${ }^{10}$. The information discussed in this paragraph are in preparation of manuscripts for publication.

NFкB can be activated by several stimuli and has ubiquitous functions such as regulating cytokine expression. Increased p65 in quiescent BCCs treated with M1 MФderived exosomes was noted for the more aggressive MDA-MB-231 cells. Such treatment was important for the M1 MФ-mediated cycling of the quiescent cells (Fig. 7). It will be of interest to study other triple negative $\mathrm{BCCs}$ to determine if the observed finding is relevant for this BCC subtype. Interestingly, TLR4 was expressed on BCCs after exposure to $\mathrm{M} 1 \mathrm{M} \Phi$-derived exosomes. This finding was of interested to begin other research to understand how dormancy $\mathrm{BC}$ might resurge into metastatic disease. As an example, TLR4 can be activated by the release of ligands when the microbiome ligands are released into the periphery. Additionally, TLR4 can be active by DAMPS. These ligands may reverse the dormant phase of BCCs perhaps partly via NFKB activation ${ }^{48}$.

In vivo, M1 MФs sensitized CSCs to carboplatin (Fig. 8a). The expression of P-gp in BCCs were similar for vehicle and those that survived carboplatin (Fig. 8b). Thus, resistance cannot be explained by P-gp. The mice injected with M1 MФs and carboplatin showed prolonged survival. We assume that the in vitro studies were inadequate-perhaps requiring additional exosomes from freshly prepared $M 1 M \Phi$, or requiring longer treatment. Alternatively, the in vivo outcome suggested that the M1 MФs might need interaction with other BM microenvironmental cells. Our unpublished data, literature and limited information on MSCs support a MSC-MФ axis to influence $\mathrm{BCC}$ behavior in BM (Fig. 1) ${ }^{49}$. In ongoing research studies, we noted distinct secretomes (soluble and vesicles) by activated and inactivated MSCs. We are currently identifying the candidate secretome(s) involved in determining how the BM microenvironment sustain a particular $М \Phi$ type.

It is interest that M1 MФ-derived exosomes induced TLR4 in BCCs (Fig. 7a). The TLR4 was not exogenously derived from the exosomes because such expression was not noted for the nontumorigenic cells (Fig. 7a). The model incorporating TLR4 and TLR3 was intended to gain insights on BC resurge. TLR4, which is a PRR, can also interact with DAMPs. Thus the incorporation of PRRs in the study is highly significant since these receptors can be activated by the microbiome secretome, which has been linked to $\mathrm{BC}^{50,51}$.

Upon synchronization, BCCs showed similar effects on cycling quiescence as the studies conducted with CSCs in the presence of $M \Phi$ s. This suggested that $M \Phi$ s may affect any $\mathrm{BCC}$ within an environment that promotes survival and reduced cycling. Thus, it is likely that dormant $\mathrm{BCCs}$ might be heterogeneous with respect to relative maturity. These findings have added to the general information on BM stromal support of $\mathrm{BC}$ survival. The data in this report have provided information that would lead to omics studies for the purpose of understanding how dormancy and cancer recurrence occur. We are in the process of comparing the exosomes from M1 and M2 $M \Phi$ s. These analyses are insufficient since the preliminary evidence suggest that proteins within the exosomes might also be responsible (Unpublished). The contents of the exosomes involved in these studies are included in an independent paper, in preparation for publication.

We have determined the MФs in healthy and tumorbearing mice using a model of dormancy and reverse dormancy $^{11}$ (Fig. 2). M2 and not M1 MФs were detected in the unactivated mice. The M2 MФs continued to be 
present during dormancy, supporting the findings in this study in which M2 MФs are shown to sustain dormancy. Reversed dormancy, which used cell-delivered miR-222/223, resulted in a switch in MФs to M1 type. Together, this study showed M2 MФs involvement in dormancy and during recurrence, the $M \Phi$ type is switched to M1 type. An unanswered question in this manuscript is the contents of the exosomes that cause the M2 MФs to switch to M1 type to reverse dormancy. We have performed next generation sequencing of the exosomes as well as singlecell sequencing to shown dedifferentiation of BCCs.

\section{Acknowledgments}

We thank Mr. Luke Fritzky and Mr. Joel Pierre for assisting with the sectioning of mouse tissues. The work was supported by an award given by the Department of Defense, W81XWH-11-1-0276 to P.R. and by the New Jersey Commission on Cancer Research (NJCCR) pre-doctoral fellowship grant \# DFHS15PPC049 to NDW. This work is in partial fulfillment for a Ph.D. thesis for N.D.W.

\section{Author details}

${ }^{1}$ Rutgers New Jersey Medical School (NJMS), Newark, NJ, USA. ${ }^{2}$ Rutgers Graduate School of Biomedical Sciences, Newark, NJ, USA. ${ }^{3}$ Dept of Pathology and Laboratory Medicine, Philadelphia, PA, USA. ${ }^{4}$ NJMS, Department of Cell Biology and Molecular Medicine, Newark, NJ, USA

\section{Conflict of interest}

The authors declare that they have no conflict of interest.

\section{Publisher's note}

Springer Nature remains neutral with regard to jurisdictional claims in published maps and institutional affiliations.

Supplementary Information accompanies this paper at (https://doi.org/ 10.1038/s41419-019-1304-z).

Received: 4 May 2018 Revised: 23 December 2018 Accepted: 2 January 2019

Published online: 25 January 2019

\section{References}

1. Sosa, M. S., Bragado, P. \& Aguirre-Ghiso, J. A. Mechanisms of disseminated cancer cell dormancy: an awakening field. Nat. Rev. Cancer 14, 611-622 (2014).

2. Braun, S. et al. A pooled analysis of bone marrow micrometastasis in breast cancer. New Engl. J. Med 353, 793-802 (2005).

3. Hosseini, $\mathrm{H}$. et al. Early dissemination seeds metastasis in breast cancer. Nature 540, 552-558 (2016).

4. Braun, S., Auer, D. \& Marth, C. The prognostic impact of bone marrow micrometastases in women with breast cancer. Cancer Invest. 27, 598-603 (2009).

5. Talmadge, J. E. Clonal selection of metastasis within the life history of a tumor. Cancer Res. 67, 11471-11475 (2007).

6. Uhr, J. W. \& Pantel, K. Controversies in clinical cancer dormancy. Proc. Nat Acad. Sci. 108, 12396-12400 (2011).

7. Giuliano, M. et al. Perspective on circulating tumor cell clusters: why it takes a village to metastasize. Cancer Res. 78, 845-852 (2018).

8. Banys, M., Hartkopf, A. D., Krawczyk, N., Becker, S. \& Fehm, T. Clinical implications of the detection of circulating tumor cells in breast cancer patients. Biomark. Med. 6, 109-118 (2012).

9. Patel, S. A. et al. Mesenchymal stem cells protect breast cancer cells through regulatory $\mathrm{T}$ cells: role of mesenchymal stem cell-derived TGF-beta. J. Immunol. 184, 5885-5894 (2010).

10. Patel, S. A. et al. Delineation of breast cancer cell hierarchy identifies the subset responsible for dormancy. Sci. Rep. 2, 906 (2012)
11. Bliss, S. A. et al. Mesenchymal stem cell-derived exosomes stimulate cycling quiescence and early breast cancer dormancy in bone marrow. Cancer Res. 76, 5832-5844 (2016)

12. Lim, P. K. et al. Gap junction-mediated import of microrna from bone marrow stromal cells can elicit cell cycle quiescence in breast cancer cells. Cancer Res. 71, 1550-1560 (2011).

13. Muller-Sieburg, C. E. \& Deryugina, E. The stromal cells' guide to the stem cell universe. Stem Cells 13, 477-486 (1995).

14. Asada, N., Takeishi, S. \& Frenette, P. S. Complexity of bone marrow hematopoietic stem cell niche. Int. J. Hematol. 106, 45-54 (2017).

15. Mills, C. D., Kincaid, K., Alt, J. M., Heilman, M. J. \& Hill, A. M. Pillars article: M-1/M-2 macrophages and the Th1/Th2 paradigm. J. Immunol. 2000. 164: 6166-6173. J. Immunol. 199, 2194-2201 (2017).

16. Arora, S., Dev, K., Agarwal, B., Das, P. \& Syed, M. A. Macrophages: their role, activation and polarization in pulmonary diseases. Immunobiology 223, 383-396 (2018)

17. Ferrante, C. J. \& Leibovich, S. J. Regulation of macrophage polarization and wound healing. Adv. Wound Care 1, 10-16 (2012).

18. Italiani, P. \& Boraschi, D. From monocytes to M1/M2 macrophages: phenotypical vs. functional differentiation. Front. Immunol. 5, 514 (2014).

19. Haricharan, S. \& Brown, P. TLR4 has a TP53-dependent dual role in regulating breast cancer cell growth. Proc. Natl Acad. Sci. 112, E3216-E3225 (2015).

20. Santos, M. F. et al. Comparative analysis of innate immune system function in metastatic breast, colorectal, and prostate cancer patients with circulating tumor cells. Exp. Mol. Pathol. 96, 367-374 (2014).

21. Vacchelli, E., Enot, D. P., Pietrocola, F., Zitvogel, L. \& Kroemer, G. Impact of pattern recognition receptors on the prognosis of breast cancer patients undergoing adjuvant chemotherapy. Cancer Res. 76, 3122-3126 (2016).

22. Midwood, K. S., Piccinini, A. M. \& Sacre, S. Targeting Toll-like receptors in autoimmunity. Curr. Drug Targets 10, 1139-1155 (2009).

23. Rameshwar, $\mathrm{P}$. et al. The dynamics of bone marrow stromal cells in the proliferation of multipotent hematopoietic progenitors by substance P: an understanding of the effects of a neurotransmitter on the differentiating hematopoietic stem cell. J. Neuroimmunol. 121, 22-31 (2001).

24. Pearlstein, E., Dienstman, S. \& Defendi, V. Identification of macrophage external membrane proteins and their possible role in cell adhesion. J. Cell. Biol. 79, 263-267 (1978).

25. Ckark, B. R. \& Keating, A. Biology of bone marrow stroma. Ann. NY Acad. Sci. 770, 70-78 (1995).

26. Mantovani, A. et al. The chemokine system in diverse forms of macrophage activation and polarization. Trends Immunol. 25, 677-686 (2004).

27. Mills, C. D., Kincaid, K., Alt, J. M., Heilman, M. J. \& Hill, A. M. M-1/M-2 macrophages and the Th1/Th2 paradigm. J. Immunol. 164, 6166-6173 (2000).

28. Stein, M., Keshav, S., Harris, N. \& Gordon, S. Interleukin 4 potently enhances murine macrophage mannose receptor activity: a marker of alternative immunologic macrophage activation. J. Exp. Med. 176, 287-292 (1992).

29. Verreck, F. A. et al. Human IL-23-producing type 1 macrophages promote but IL-10-producing type 2 macrophages subvert immunity to (myco) bacteria. Proc. Natl Acad. Sci. 101, 4560-4565 (2004).

30. M, S. In Cellular Components of Stroma In Vivo In Comparison Within Vitro Systems (ed Tavassoli M) Chapter 9 (1989).

31. Yu, V. W. \& Scadden, D. T. Heterogeneity of the bone marrow niche. Curr. Opin. Hematol. 23, 331-338 (2016)

32. Loegl, J. et al. Hofbauer cells of M2a, M2b and M2c polarization may regulate feto-placental angiogenesis. Reproduction 152, 447-455 (2016).

33. Bisgaard, L. S. et al. Bone marrow-derived and peritoneal macrophages have different inflammatory response to oxLDL and M1/M2 marker expressionimplications for atherosclerosis research. Sci. Rep. 6, 35234 (2016).

34. Vijayan, $V$. et al. A new immunomodulatory role for peroxisomes in macrophages activated by the TLR4 ligand lipopolysaccharide. J.Immunol. 198, 2414-2425 (2017)

35. Teixeira-Coelho, M. et al. Differential post-transcriptional regulation of $\mathrm{LL}-10$ by TLR2 and TLR4-activated macrophages. Eur. J. Immunol. 44, 856-866 (2014).

36. Fabriek, B. O. et al. The macrophage scavenger receptor CD163 functions as an innate immune sensor for bacteria. Blood 113, 887-892 (2009).

37. Sangiorgi, B. \& Panepucci, R. A. Modulation of immunoregulatory properties of mesenchymal stromal cells by Toll-like receptors: potential applications on GVHD. Stem Cells Int. 2016, 9434250 (2016).

38. Self-Fordham, J. B., Naqvi, A. R., Uttamani, J. R., Kulkarni, V. \& Nares, S. MicroRNA dynamic regulators of macrophage polarization and plasticity. Front. Immunol. 8, 1062 (2017). 
39. Hayden, M. S. \& Ghosh, S. Shared principles in NF-KB signaling. Cell 132, 344-362 (2008).

40. Shostak, K. \& Chariot, A. NF-KB, stem cells and breast cancer: the links get stronger. Breast Cancer Res. 13, 214 (2011).

41. Smith, S. M., Lyu, Y. L. \& Cai, L. NF-KB affects proliferation and invasiveness of breast cancer cells by regulating CD44 expression. PLoS One $\mathbf{9}$, e106966 (2014).

42. Mattioli, I. et al. Transient and selective NF-kappa B p65 serine 536 phosphorylation induced by $\mathrm{T}$ cell costimulation is mediated by I kappa B kinase beta and controls the kinetics of p65 nuclear import. J. Immunol. 172, 6336-6344 (2004).

43. Cuzzocrea, S. et al. Pyrrolidine dithiocarbamate attenuates the development of acute and chronic inflammation. Br. J. Pharmacol. 135, 496-510 (2002).

44. Karagiannis, G. S. et al. Neoadjuvant chemotherapy induces breast cancer metastasis through a TMEM-mediated mechanism. Sci. Transl. Med 9, 399 (2017).

45. Moharita, A. L. et al. SDF-1a regulation in breast cancer cells contacting bone marrow stroma is critical for normal hematopoiesis. Blood 108, 3245-3252 (2006).
46. Tsai, J. H. \& Yang, J. Epithelial-mesenchymal plasticity in carcinoma metastasis, Genes Dev. 27, 2192-2206 (2013).

47. Yang, M., Ma, B., Shao, H., Clark, A. M. \& Wells, A. Macrophage phenotypic subtypes diametrically regulate epithelial-mesenchymal plasticity in breast cancer cells. BMC Cancer 16, 419 (2016).

48. Jin, J. et al. Coactivation of TLR4 and TLR2/6 coordinates an additive augmentation on IL-6 gene transcription via p38MAPK pathway in U937 mononuclear cells. Mol. Immunol. 49, 423-432 (2011).

49. Geng, L. X., Yin, X. H., Li, B. \& Ding, S. F. Inhibition of M1 macrophage specific gene expression and promotion of M2 macrophage specific gene expression by bone mesenchymal stem cells. Int. J. Clin. Pathol. 10, 750-759 (2017).

50. Ahmed, A., Redmond, H. P. \& Wang, J. H. Links between Toll-like receptor 4 and breast cancer. Oncoimmunology 2, e22945 (2013).

51. Nabet, B. Y. et al. Exosome RNA unshielding couples stromal activation to pattern recognition receptor signaling in cancer. Cell 170, 352-366.e313 (2017). 